\title{
EDITORIAL
}

nature

cell biology

\section{A new dawn in the land of the rising sun}

Although Japan is long established as a major player in most natural sciences disciplines, arguably its research potential has been held back by a dated system of academic appointments. The Japanese academic landscape is characterized by an overly pyramidal distribution of seniority, where some professors manage to build up huge research empires. Although efficient in terms of overall research output, this arrangement runs the risk of stifling young researchers in the prime of their careers and there is a sizeable imbalance between the number of scientists in training and the academic positions ultimately available to them. Laudably, overseas research experience is valued in Japan and ambitious young researchers still aim for a postdoc in a top US or European lab in the hope of leapfrogging the queue for the few available independent research positions. Maybe the low ratio of postdocs to graduate students is due to the sizeable Japanese postdoc population overseas and since reciprocal visits by foreign researchers to Japan remain all too infrequent. The consequence is that a postdoc in Japan is often characterized by uncertain career progression and a high teaching burden. As a result, some of the best researchers stay overseas - at least until they can return to a full professorship. As a corollary, this system makes Japan less attractive to foreign researchers.

However, although the academic system in Japan may appear old fashioned, it is not that different from many European countries. In Germany, steps have been taken to provide an alternative fast-track path to independent research positions that circumvents the traditional cumbersome route to a professorship. In an effort to bypass the parochialism of this system and to open the prospect of independent research to younger scientists, the German government instigated the 'junior professorship' a few years ago. We commented on the shortcomings of this scheme previously (Nature Cell Biol. 6, 793; 2004).

Although continental Europe shows few signs of a bona fide change in academic career structure, in Japan at least there are signs of significant change: the '21st century COE' (centers of excellence) programme was launched in 2002 by MEXT (the ministry responsible for science and higher education). The COE programme is unique in Japan in several respects: first, it allows universities to look beyond department and faculty boundaries to develop graduate programmes and collaborative research based on scientific synergism, rather than administrative structures. Second, COE programmes do not merely provide research funds, but budget for graduate and postgraduate education. This is revolutionary in Japan, where students essentially have had to provide their own funds, not just for living expenses, but also for conference fees and travel. Third, it is among the first programme grants awarded based on open competition. Finally, COE funds are largely administered by researchers, providing them with the freedom to support research without administrative red tape. One outcome is that many COEs have appointed independent junior faculty members.

One COE, the University of Tokyo's 'Center for integrated brain medical science', ran a retreat in the foothills of Mount Fuji in July, which exemplified the programme's support of graduates and postgraduates, as well as the successful interfacing of multiple disciplines ranging from systems neurobiology to signal transduction. Haruhiko Bito, (Department of Neurochemistry and COE member) is enthusiastic - "I believe that the COE programme has been one of the finest inventions to deregulate research and education in Japanese universities. It promotes creative research programmes that go beyond the border of conventional departments, and a remarkable flexibility was introduced in bringing research support to students and independent research units for young faculty". Although support for the COE programme is high, there is widespread anxiety as MEXT is set to relaunch a revised 'Global COE' programme next year, in time for the first renewals, with a requested budget of 23.1 billion Yen (197 million US\$) in 2007. There will be a bigger focus on collaborative research beyond Japan and on the provision of independent research by young investigators. The ministry plans a tough round of re-evaluations and the total number of COEs is set to reduce from the current 274 awarded between 2002 and 2004, to 150 that will be awarded between 2007 and 2011 (60 in 2007). Although MEXT emphasises that as many grants as possible will be renewed, there will be slots for new proposals.

More recently, MEXT has also launched a separate programme for 2007 with a self explanatory title that translates as "a programme to promote the improvement of the research environment for young researchers to be independent". The programme, which is currently limited to only nine universities, provides promising scientists in their thirties with independence and resources to channel their energies into establishing new research programmes at a highly productive point of their careers. In contrast to Germany's 'junior professors' the programme's tenure track seems to provide long-term perspective. Immunologist Akihiko Yoshimura of Kyushu University comments that "the MEXT program is very important because it may become a trigger of reformation of the entire Japanese academic career system. Research suggests that most great discoveries are made at age 30-40. Therefore making PIs at an age less than 35 is reasonable" and he voted in favour of establishing 16 such positions at his faculty (known as the 'superstar programme'). However, he cautions that this programme will be a big challenge and points out that it remains unclear how much pressure there will be on grant recipients to secure independent secondary funding and just how competitive small labs will be in the current research environment. Yoshimura states that it remains unclear how his university will support tenured 'superstar' researchers, as no additional positions have been earmarked. Indeed, concern that these new positions will ultimately put pressure on existing faculty was the main reason why many opted out of the programme. It will be important to reassure academics that these start-up positions are meant to move towards university professorships with administrative duties and teaching responsibilities, as otherwise a two-track system may develop that will not necessarily encourage a harmonious research environment at universities. 